Journal of Mathematical Sciences, Vol. 195, No. 4, December, 2013

\title{
A NOTE ON BARELY TRANSITIVE GROUPS
}

\section{Kuzucuoğlu}

UDC 512.54

Abstract. We study some properties for barely transitive group.

A finite group satisfying the normalizer condition is nilpotent. The locally finite groups satisfying the normalizer condition with trivial center is constructed by Heineken and Mohammad. The study of barely transitive groups began with these examples and the examples constructed by Hartley. Recall that a group is called a barely transitive group if it acts on an infinite set transitively and faithfully, and every orbit of every proper subgroup is finite. An infinite group $G$ is called a quasi-finite group (or Schmidt group) if all of its proper subgroups are finite. One can easily see that an infinite group $G$ is barely transitive in its regular permutation representation if and only if $G$ is a quasi-finite group. For this reason, there is a close structural relation between barely transitive groups and quasi-finite groups. One can see that Olshanskii groups of exponent $p$, for a fixed prime $p$, given in [3] are primitive barely transitive groups. These Olshanskii groups are simple. Indeed, for every normal subgroup $N$ of a transitive group $G$, every $N$-orbit is a $G$-block. Therefore, any primitive barely transitive group is simple. It is known that a locally finite barely transitive group cannot be primitive [2]. For the primitivity of an arbitrary barely transitive group, we have the following:

Theorem 1 (see [1]). A barely transitive group is totally imprimitive if and only if it is locally graded.

Recall that a group $G$ is called locally graded if every finitely generated subgroup has a proper subgroup of finite index.

Theorem 2 (see [1]). Let $G$ be a barely transitive group. If there exists $x \in G$ satisfying for any nonidentity $y$ in $G,\langle x\rangle \cap\langle y\rangle \neq 1$, then $G$ is isomorphic to one of the followings:

(1) $C_{p^{\infty}}$ for some prime $p$;

(2) $G$ is a finitely generated quasi-finite group with a trivial point stabilizer, $Z(G) \neq 1$ and $G / Z(G)$ is simple, and finitely many primes divides the order of elements of $G$.

In particular, such a group $G$ cannot be torsion free.

Examples of groups in item (2) exist by construction of Ol'shanskii [3].

\section{REFERENCES}

1. C. Betin and M. Kuzucuoğlu, "On locally graded barely transitive groups" (submitted).

2. M. Kuzucuoğlu, "Barely transitive permutation groups," Arch. Math., 55, 521-532 (1990).

3. A. Yu. Ol'shanskii, Geometry of Defining Relations in Groups, Kluwer Academic (1991).

M. Kuzucuoğlu

Department of Mathematics, Middle East Technical University, Ankara, Turkey

E-mail: matmah@metu.edu.tr

Translated from Sovremennaya Matematika i Ee Prilozheniya (Contemporary Mathematics and Its Applications), Vol. 83, Modern Algebra and Its Applications, 2012. 